\title{
(6) OPEN ACCESS \\ Prevalence of comorbidities in rheumatoid arthritis and evaluation of their monitoring: results of an international, cross-sectional study (COMORA)
}

\author{
Maxime Dougados, ${ }^{1,2}$ Martin Soubrier, ${ }^{3}$ Anna Antunez, ${ }_{1}^{4}$ Peter Balint, ${ }_{1}^{5}$ \\ Alejandro Balsa, ${ }^{6}$ Maya H Buch, ${ }^{7,8}$ Gustavo Casado, ${ }^{9}$ Jacqueline Detert, ${ }^{10}$ \\ Bassel El-zorkany, ${ }^{11}$ Paul Emery, ${ }^{7,8}$ Najia Hajjaj-Hassouni, ${ }^{12}$ Masayoshi Harigai, ${ }^{13}$ \\ Shue-Fen Luo, ${ }^{14}$ Reka Kurucz, ${ }^{5}$ Gabriel Maciel, ${ }^{15}$ Emilio Martin Mola, ${ }^{16}$ Carlo \\ Maurizio Montecucco, ${ }^{17}$ lain McInnes, ${ }^{18}$ Helga Radner, ${ }^{19}$ Josef S Smolen, ${ }^{19}$ \\ Yeong-Wook Song, ${ }^{20}$ Harald Erwin Vonkeman, ${ }^{21}$ Kevin Winthrop, ${ }^{22}$ Jonathan Kay ${ }^{23}$
}

\begin{abstract}
Handling editor Tore K Kvien
- Additional material is published online only. To view please visit the journal online (http://dx.doi.org/10.1136/ annrheumdis-2013-204223).

For numbered affiliations see end of article.
\end{abstract}

\section{Correspondence to} Professor Maxime Dougados, Rhumatologie B, Hôpital Cochin, 27 rue du Fg SaintJacques, Paris 75014, France; maxime.dougados@cch.aphp.fr

Received 10 July 2013 Revised 26 August 2013 Accepted 8 September 2013 Published Online First 4 October 2013

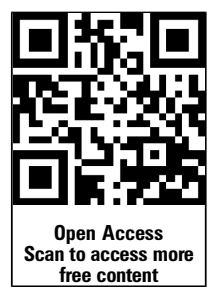

\begin{tabular}{|l|}
\hline To cite: Dougados M, \\
Soubrier M, Antunez A, \\
et al. Ann Rheum Dis \\
2014;73:62-68.
\end{tabular}

\section{ABSTRACT}

Background Patients with rheumatoid arthritis (RA) are at increased risk of developing comorbid conditions.

Objectives To evaluate the prevalence of comorbidities and compare their management in RA patients from different countries worldwide.

Methods Study design: international, cross-sectional. Patients: consecutive RA patients. Data collected: demographics, disease characteristics (activity, severity, treatment), comorbidities (cardiovascular, infections, cancer, gastrointestinal, pulmonary, osteoporosis and psychiatric disorders).

Results of 4586 patients recruited in 17 participating countries, 3920 were analysed (age, 56 \pm 13 years; disease duration, $10 \pm 9$ years (mean \pm SD); female gender, 82\%; DAS28 (Disease Activity Score using 28 joints)-erythrocyte sedimentation rate, 3.7 1.6 (mean \pm SD); Health Assessment Questionnaire, 1.0 \pm 0.7 (mean \pm SD); past or current methotrexate use, $89 \%$; past or current use of biological agents, 39\%. The most frequently associated diseases (past or current) were: depression, 15\%; asthma, 6.6\%; cardiovascular events (myocardial infarction, stroke), 6\%; solid malignancies (excluding basal cell carcinoma), 4.5\%; chronic obstructive pulmonary disease, $3.5 \%$. High intercountry variability was observed for both the prevalence of comorbidities and the proportion of subjects complying with recommendations for preventing and managing comorbidities. The systematic evaluation of comorbidities in this study detected abnormalities in vital signs, such as elevated blood pressure in $11.2 \%$, and identified conditions that manifest as laboratory test abnormalities, such as hyperglycaemia in 3.3\% and hyperlipidaemia in $8.3 \%$.

Conclusions Among RA patients, there is a high prevalence of comorbidities and their risk factors. In this multinational sample, variability among countries was wide, not only in prevalence but also in compliance with recommendations for preventing and managing these comorbidities. Systematic measurement of vital signs and laboratory testing detects otherwise unrecognised comorbid conditions.

\section{INTRODUCTION}

The long-term prognosis of rheumatoid arthritis (RA) has improved dramatically following the introduction of highly effective medications, such as methotrexate leflunomide and biological agents, ${ }^{1} 2$ and as the result of close monitoring and regular adjustment of treatment to the targets of low disease activity or remission. ${ }^{3}$ However, comorbidities may shorten the life span of patients with RA. ${ }^{4-6}$ This higher death rate appears to be the consequence of an increased prevalence of cardiovascular disease, a greater incidence of infections, and the development of certain malignancies in patients with RA. ${ }^{7-11}$ Also, osteoporotic fractures are more commonly observed in patients with RA and significantly affect the prognosis for functional decline. ${ }^{12} 13$ In addition, RA patients with more comorbidities experience greater functional impairment. ${ }^{14}$

Some of these comorbidities are observed more often among RA patients because of the medications with which they are treated, especially glucocorticoids, ${ }^{10}$ and because of traditional risk factors, such as tobacco smoking. ${ }^{15}$ However, chronically active inflammation also predisposes to the development of these comorbidities. ${ }^{16}$

Unfortunately, comorbidities are not well managed in RA patients. ${ }^{17-20}$ To address this disparity, the European League Against Rheumatism (EULAR) proposed specific recommendations for detecting and managing specific comorbidities and preventing their development when possible. These include recommendations that all patients with RA should be vaccinated against influenza every year and against pneumococci every 5 years ${ }^{21}$ and should be evaluated for cardiovascular risk annually. Because chronically active inflammation contributes to the development of cardiovascular disease, these recommendations suggest that the cardiovascular risk score be multiplied by a factor of 1.5 when two of the following three criteria are met: (1) disease duration longer than 10 years; (2) presence of circulating rheumatoid factor or anti-citrullinated protein antibodies; (3) presence of extra-articular manifestations. ${ }^{22}$ 
The COMORA (COMOrbidities in Rheumatoid Arthritis) Study had two major objectives. The first was to evaluate variability in the prevalence of comorbidities and their risk factors between participating countries. The second was to assess whether there is a disparity between existing national recommendations and the actions implemented in daily clinical practice to detect and prevent the development of these comorbidities.

\section{PATIENTS AND METHODS \\ Study design}

This was a cross-sectional, observational, multicentre, international study.

\section{Patient recruitment}

The scientific committee chose national principal investigators for this study. Their task was to select rheumatologists who would be representative of their country and to conduct the study in accordance with good clinical practice. The protocol was reviewed and approved by all local institutional review boards or ethics committees. Consecutive patients visiting the participating rheumatologists were invited to enrol in the study if they were at least 18 years of age, fulfilled the 1987 American College of Rheumatology classification criteria for $\mathrm{RA},{ }^{23}$ and were able to understand and complete the questionnaires that were administered. Written informed consent was obtained from all subjects before enrolment.

\section{Sample size}

The sample size calculation was based on the precision (width) of the 95\% CI of the proportions of expected events (eg, prevalence of each comorbidity). For example, it was calculated that a sample of 4000 patients would allow the $35 \%$ prevalence of a given comorbidity, $\mathrm{X}$, to be estimated with a precision of $1.5 \%$ (95\% CI $33.5 \%$ to $36.5 \%$ ), or the $1 \%$ prevalence of another comorbidity, Y, to be estimated with a precision of $0.3 \%(95 \%$ CI $0.7 \%$ to $1.3 \%$ ).

Investigators in each participating country were expected to enrol at least 200 patients.

\section{Data collected}

A case report form specifically created for this study was used to collect four categories of data.

1. Characteristics of demographics and the disease. Patients' demographic characteristics included: age, gender, body mass index, smoking status, alcohol intake, marital status, socioeconomic status and highest level of education completed. Disease activity was assessed using the DAS28 (Disease Activity Score using 28 joints)-erythrocyte sedimentation rate (ESR) ${ }^{24}$ and the C-reactive protein level. Disease severity was evaluated from the history of joint surgery to address structural damage caused by RA (eg, total joint arthroplasty, arthrodesis, metacarpophalangeal or metatarsophalangeal joint resections). Past and current medications used to treat RA were also recorded, including non-steroidal anti-inflammatory drugs, corticosteroids and conventional and biological disease-modifying antirheumatic drugs (DMARDs).

2. History or current evidence of comorbidities. Ischaemic cardiovascular disease (myocardial infarction, stroke), cancers (colon, skin, lung, breast and uterus for women, prostate for men) and lymphoma, gastrointestinal diseases (diverticulitis, ulcers), infections (hepatitis), lung disease (chronic obstructive pulmonary disease (COPD), asthma) and psychiatric disorders (depression).
3. Coexisting risk factors. Risk factors for cardiovascular diseases (hypertension, diabetes, dyslipidaemia, family history of myocardial infarction or sudden death), risk factors for infectious diseases and vaccination status, risk factors for cancers (family history of prostate, breast or colon cancer; adenomatosus polyposis and/or personal history of inflammatory bowel disease (for colon cancer) and history of numerous ( $>40$ ) nevi for skin cancer).

4. Compliance with current national recommendations regarding management (prevention, detection and treatment) of these comorbidities. For example, annual estimation of cardiovascular risk.

For each patient, information was gathered by a study investigator during a face-to-face interview at a dedicated study visit and through review of the medical record.

\section{Data analysis}

The first step of the analysis was to describe the baseline characteristics of the enrolled patients, by country, including the prevalence of each comorbidity and associated disease risk factors (\% and $95 \% \mathrm{CI}$ ).

To estimate any disparity that might exist between published recommendations and daily clinical practice in the prevention, detection and management of these comorbidities, the percentage of patients monitored and managed according to national guidelines was calculated. The definition of 'optimal' management for the evaluated comorbidities was primarily based on recommendations made by international scientific societies ${ }^{17} 18$ and/or national healthcare systems ${ }^{25}$ and/or the recommendations of the French Society of Rheumatology to prevent, detect and control comorbidities in patients with inflammatory rheumatic diseases. ${ }^{26}$

For cardiovascular diseases, a patient was considered to be optimally monitored when risk factors for cardiovascular events (eg, blood pressure, blood glucose level, low-density lipoprotein (LDL) cholesterol level) were evaluated annually. Patients older than 50 years were considered to be managed optimally if they were receiving an antithrombotic drug, in the setting of a past thrombotic cardiovascular event, or if their Framingham Risk Score $^{27}$ was calculated to be $20 \%$ or more above the upper limit of normal after being adjusted for RA (multiplied by a factor of $1.5)$, in the presence of specific RA characteristics. ${ }^{22}$ Finally, we evaluated the proportion of patients in whom the systematic evaluation of risk factors for cardiovascular diseases during the conduct of the study detected hypertension (eg, systolic pressure $>140 \mathrm{~mm} \mathrm{Hg}$ or diastolic pressure $>80 \mathrm{~mm} \mathrm{Hg}$ or $>130 \mathrm{~mm} \mathrm{Hg}$ and $70 \mathrm{~mm} \mathrm{Hg}$, respectively, in the setting of concomitant diabetes mellitus ${ }^{26}$ ), elevated LDL cholesterol (above the targeted value defined with regard to the number of concomitant additional cardiovascular risk factors ${ }^{28}$ ) and hyperglycaemia (random blood glucose level $>1.26 \mathrm{~g} / \mathrm{L}^{28}$ ).

A patient was considered to be monitored optimally for infectious diseases if he or she had had (1) a dental examination within the previous year, (2) an influenza vaccination within the previous year, and (3) a pneumococcal vaccination within the previous 5 years.

A patient was considered to be monitored optimally for cancer if age- and sex-appropriate cancer screening recommendations for the general population were followed. A male patient without known prostate cancer was considered to have been screened optimally for prostate cancer if a digital rectal examination and prostate-specific antigen (PSA) level had been performed between the ages of 50 and 75 years (or between the ages of 45 and 75 years for patients of African ancestry) or with at least two first-degree relatives who had prostate cancer. Subsequently, this 
evaluation had to have been repeated every 3 years for those with PSA $<1 \mathrm{ng} / \mathrm{mL}$ and annually for those with PSA between 1 and $4 \mathrm{ng} / \mathrm{mL}$. For men with PSA $>4 \mathrm{ng} / \mathrm{mL}$, evaluation by an urologist was required for the patient to be considered to have been monitored optimally. ${ }^{28}$ For breast cancer detection, a woman between the ages of 50 and 74 years without known breast cancer was considered to have been screened optimally if a mammogram had been performed within 2 years of the study visit. ${ }^{28}$ For uterine cancer detection, a woman between the ages of 25 and 65 years without known uterine cancer was considered to have been monitored optimally if a Papanicolaou smear of the cervix had been performed within 3 years of the study visit. ${ }^{28}$ For colon cancer screening, a patient over 50 years old without known colon cancer was considered to have been optimally monitored if stool had been tested for occult blood and at least one colonoscopy had been performed. For those patients at high risk of developing colon cancer (eg, those with inflammatory bowel disease or with at least two first-degree relatives who had colon or rectal cancer or at least one first-degree relative with adenomatous polyposis or with Lynch syndrome), a colonoscopy had to have been performed in the 2 years before the study visit for a patient to be considered to have been optimally monitored. ${ }^{28}$ For skin cancer detection, a patient was considered to be optimally monitored if he or she had been examined at least once by a dermatologist; if more than 40 nevi were present, annual evaluation by a dermatologist was required for optimal monitoring. ${ }^{28}$ For lung cancer screening, a patient was considered to have been monitored optimally if a chest radiograph had been performed after the onset of RA. ${ }^{26}$

A patient was considered to have been screened optimally for osteoporosis if at least one bone densitometry study had been performed after the onset of RA and if he or she was taking vitamin $\mathrm{D}$ supplementation at the time of the study visit. ${ }^{26}$

\section{RESULTS}

\section{Patients and study course}

A total of 4586 patients were recruited by investigators in the 17 participating countries between 2011 and 2012. Because a disproportionately high number of subjects were enrolled in South Korea $(n=1052)$ compared with each of the other 16 countries,
400 patients from South Korea were randomly selected for inclusion in the analysis. Fourteen patients from a single centre were excluded from the current analysis because of too many missing data, leaving a total of 3920 patients for further evaluation.

The baseline characteristics are summarised in table 1 . There was enormous intercountry variability for some characteristics: patients in North Africa tended to have more active and more severe disease, and fewer patients in some South American countries had been treated with biological agents. Detailed comparisons of the baseline characteristics for each individual country are provided in online supplementary tables S1 and S2.

\section{Prevalence of comorbidities}

The prevalence of those comorbidities that were evaluated is depicted in figure 1. Depression (past or current symptoms) was the most commonly observed comorbidity (mean 15.0\%, 95\% CI $13.8 \%$ to $16.1 \%$ ); however, the prevalence of depression varied widely among countries (from $2 \%$ in Morocco to $33 \%$ in the USA).

There was a history of ischaemic cardiovascular disease (myocardial infarction or stroke) in $6.0 \%$ (95\% CI 5.3\% to $6.8 \%$ ) of the patients. This prevalence ranged from a low of $1 \%$ in Morocco to a high of $17 \%$ in Hungary. A history of any solid tumour, excluding basal cell skin cancers, was found in $4.5 \%$ (95\% CI 3.9\% to 5.2\%) of the patients and ranged from a low of $0.3 \%$ in Egypt to a high of $12.5 \%$ in the USA). Hepatitis B infection was observed more frequently in Italy (9\%) and Taiwan $(7 \%)$ than in other countries $(2.8 \%$ (95\% CI $2.3 \%$ to $3.3 \%)$ ). The prevalence of hepatitis $\mathrm{C}$ infection was highest in Italy (6.6\%), Egypt (6.8\%) and Taiwan (4.8\%). The overall prevalence of past or present gastrointestinal ulcer was $10.8 \%(95 \% \mathrm{CI}$ $9.8 \%$ to $11.8 \%$ ). This ranged from a low of 1\% in Morocco to a high of 22\% in Egypt. Episodes of diverticulitis that had required surgical intervention were rarely observed $(0.4 \%$ (95\% CI $0.2 \%$ to $0.6 \%)$ ). Pulmonary diseases, especially COPD, were observed less commonly in Asian countries (Japan, 1.4\%; Korea, 1.3\%; Taiwan, $0.3 \%$ ) than in European countries or the USA (Hungary, $8.0 \%$; USA, $7.5 \%$ ). Detailed listings by country of the prevalence of the various comorbidities, grouped by category, are presented in online supplementary tables S3-S7.

Table 1 Baseline patient and disease characteristics of the 3920 analysed patients enrolled in the COMORA Study

\begin{tabular}{|c|c|c|}
\hline \multirow[b]{2}{*}{ Variable } & \multicolumn{2}{|l|}{ Results } \\
\hline & Global results & Extremes (countries) \\
\hline Number & 3920 & From 30 (Uruguay) to 411 (France) \\
\hline Female gender (\%) & 81.7 & From 66 (Netherlands) to 91 (Venezuela) \\
\hline Age (years), mean $\pm S D$ & $56 \pm 13$ & From 48 (Morocco/Egypt) to 63 (Japan) \\
\hline Smoking status ( $\%$ current smokers) & 13.2 & From 0.9 (Morocco) to 48 (Austria) \\
\hline Educational level (\% university or graduate school) & 24.5 & From 5.3 (Italy) to 75 (Netherlands) \\
\hline Marital status ( $\%$ married) & 69.7 & From 50 (Venezuela) to 86 (Netherlands) \\
\hline BMI (\% overweight or obese) & 50.7 & From 0 (Netherlands) to 69 (USA) \\
\hline Work status (\% currently employed) & 31.4 & From 16 (Morocco) to 46 (USA) \\
\hline Disease duration (years), mean $\pm S D$ & $9.6 \pm 8.7$ & From 7 (Morocco) to 14 (France) \\
\hline DAS28-ESR, mean \pm SD & $3.7 \pm 1.6$ & From 2.6 (Netherlands) to 5.3 ( Egypt) \\
\hline $\mathrm{HAQ}$, mean $\pm \mathrm{SD}$ & $1.0 \pm 0.7$ & From 0.7 (Taiwan) to 1.5 (Morocco) \\
\hline Prednisone (\% currently taking) & 54.3 & From 9 (UK) to 82 (Morocco) \\
\hline NSAID use ( $\%$ having taken dose during previous 3 months) & 55.2 & From 25 (Morocco) to 94 (Taiwan) \\
\hline MTX (\% ever treated) & 88.6 & From 79 (Italy) to 98 (UK) \\
\hline Any biological therapy (\% ever treated) & 38.9 & From 3 (Uruguay) to 77 (UK) \\
\hline
\end{tabular}

BMI, body mass index; DAS28-ESR, Disease Activity Score using 28 joints-erythrocyte sedimentation rate; HAQ, Health Assessment Questionnaire; MTX, methotrexate; NSAID, non-steroidal anti-inflammatory drug. 
Figure 1 Prevalence of evaluated comorbidities in the 3920 patients with rheumatoid arthritis. COPD, chronic obstructive pulmonary disease.

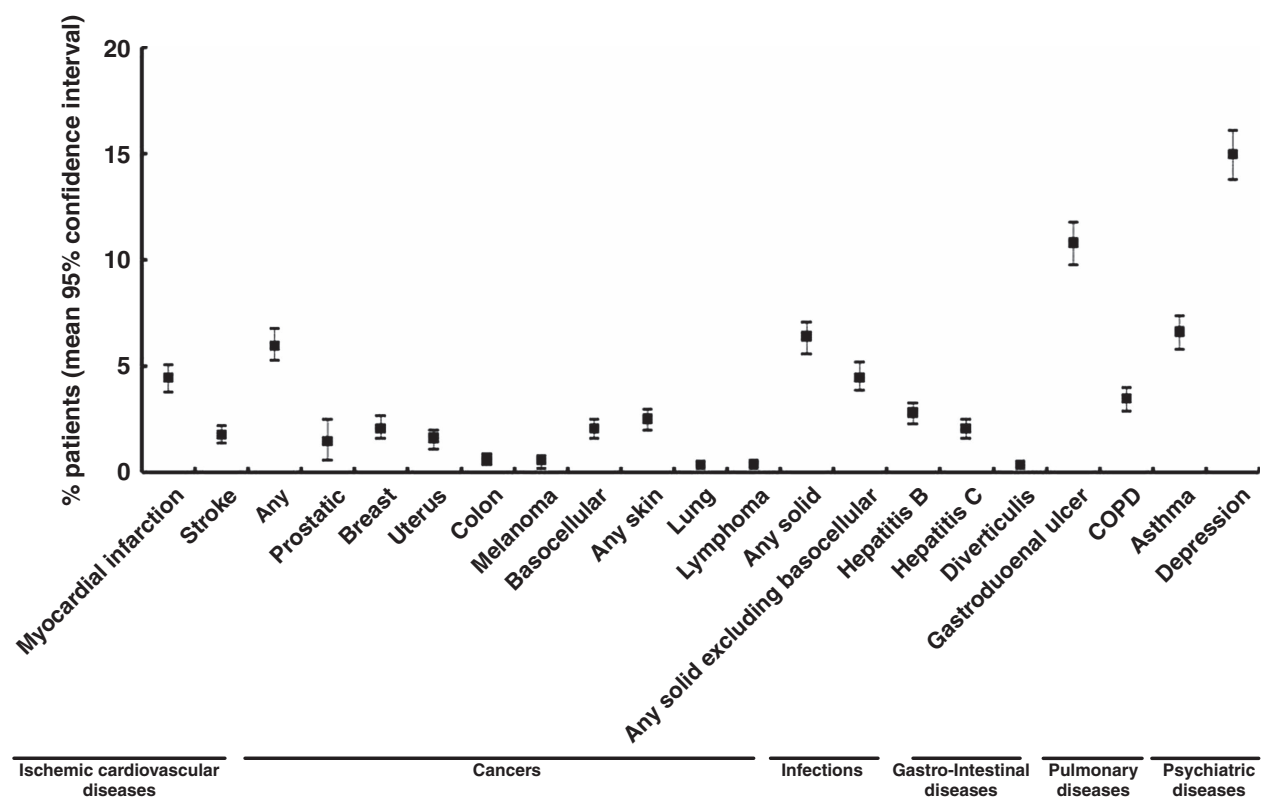

\section{Prevalence of risk factors for comorbidities}

The prevalence of various risk factors for cardiovascular disease and several malignancies is depicted in figure 2. As might be expected, given the increased prevalence of cardiovascular disease associated with RA, the most prevalent risk factors were those that predispose to cardiovascular disease, such as increased Framingham Risk Score $(42.8 \%$ (95\% CI 41.2\% to $44.3 \%)$ ), hypertension (40.4\% (95\% CI $38.9 \%$ to $41.9 \%)$ ) and hypercholesterolaemia (31.7\% (95\% CI $30.3 \%$ to $33.2 \%)$ ). As with the prevalence of comorbidities, there was considerable intercountry variability in the prevalence of risk factors. For example, the prevalence of smoking ranged from $3 \%$ in Morocco to $48 \%$ in Austria. Detailed listings by country of the prevalence of the various risk factors, grouped by comorbidity, are presented in online supplementary tables S8 and S9.

\section{Management of comorbidities \\ Cardiovascular diseases}

Annual evaluation of cardiovascular risk, including measurement of blood pressure, total serum cholesterol (high-density lipoprotein (HDL) and LDL), blood glucose and serum creatinine, was performed in 59.4\% (95\% CI 57.9\% to 60.9\%) of the patients. Of the 236 patients who had a prior myocardial infarction or stroke, $162(68.6 \%)$ were currently receiving an antithrombotic drug, but 74 (31.4\%) were not. Among the other 3684 patients who had no history of myocardial infarction or stroke, 366 would appropriately have been given prophylactic antithrombotic drug treatment because they were older than 50 years and had a calculated Framingham Risk Score above 20\%; however, of these, 299 were not receiving any antithrombotic agent. Thus, $373(9.5 \%)$ of the total number of patients

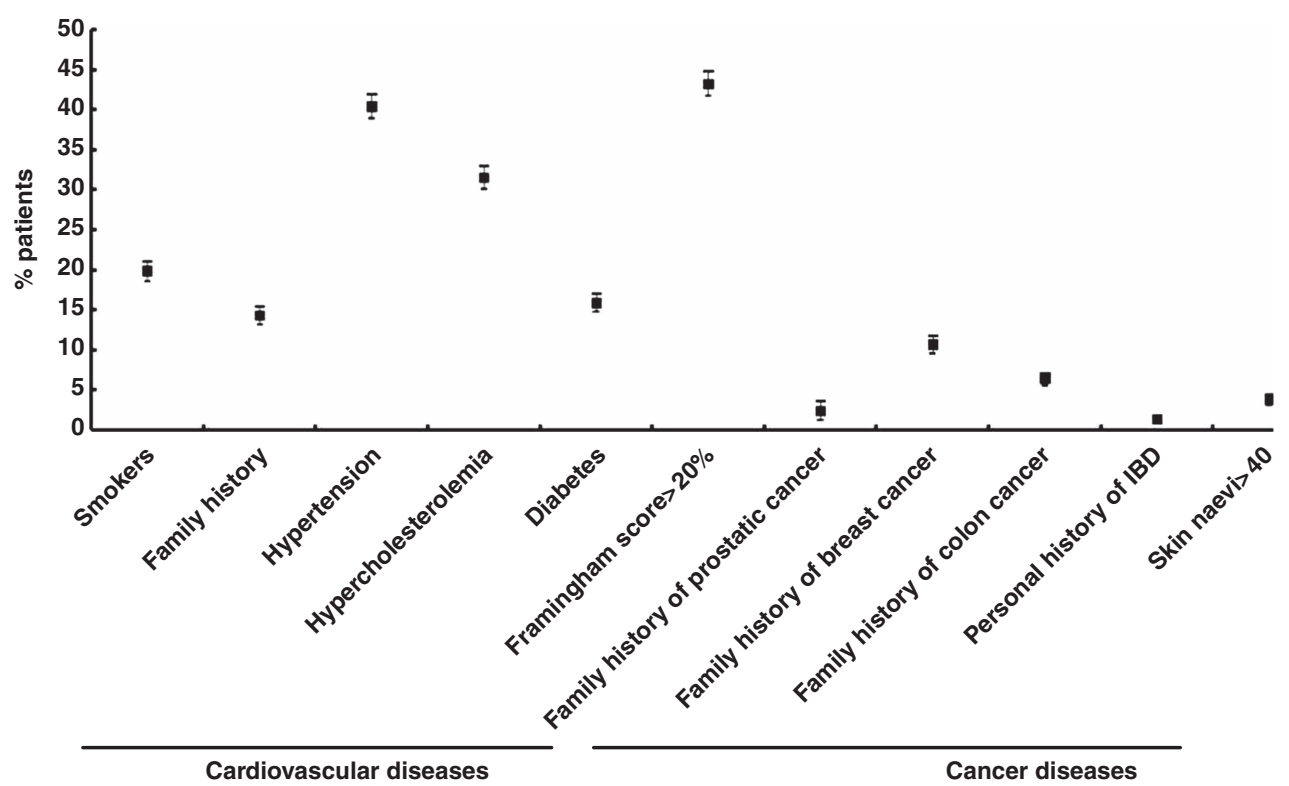

Figure 2 Prevalence of risk factors for cardiovascular and cancer diseases in the 3920 patients with rheumatoid arthritis. IBD, inflammatory bowel disease. 


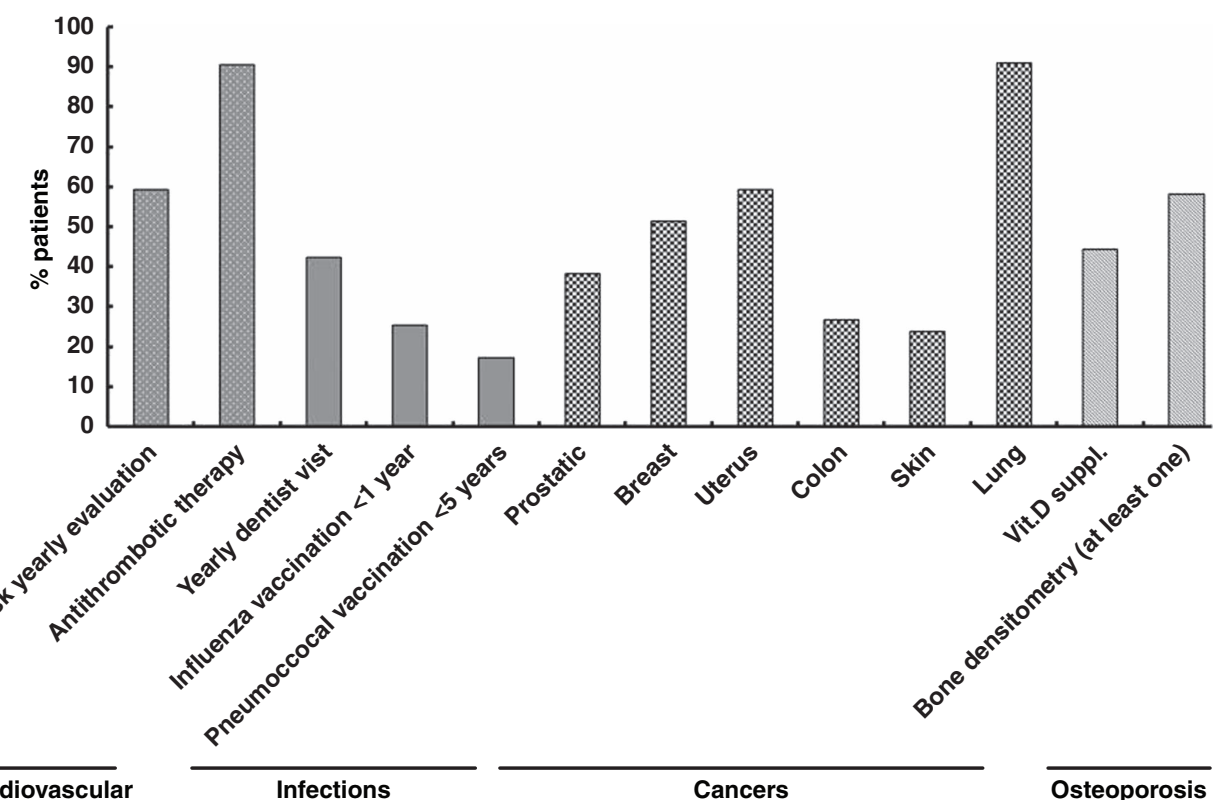

Figure 3 Percentage of patients optimally monitored with respect to some comorbidities. Vit.D suppl., vitamin D supplementation.

enrolled in this study should have been treated with antithrombotic drug prophylaxis, but were not being managed optimally to prevent cardiovascular events (figure 3 ).

The systematic assessment of certain cardiovascular risk factors in this study allowed their detection in previously undiagnosed patients. Among the 2489 patients without known hypertension, elevated blood pressure was detected in 454 $(18 \%)$. An elevated blood glucose level was detected in 131 (3.7\%) of the 3522 patients without previously diagnosed diabetes mellitus. An LDL cholesterol level above the optimal target was detected in $325(11.0 \%)$ of the 2966 patients not previously diagnosed to have a dyslipidaemia or not receiving lipid-lowering therapy.

\section{Infectious diseases}

During the year before the study visit, $42.3 \%$ (95\% CI $40.8 \%$ to $43.9 \%$ ) of all 3920 enrolled patients had undergone a dental examination. However, fewer patients were vaccinated in accordance with current recommendations: an influenza vaccination had been performed during the year before the study visit in only $938(25.3 \%(95 \%$ CI $23.9 \%$ to $26.7 \%))$ of the patients and a pneumococcal vaccination had been performed within 5 years of the study visit in only $636(17.2 \%$ (95\% CI $16.0 \%$ to $18.4 \%)$ ) of the patients. Both an influenza and a pneumococcal vaccination were performed according to current recommendations in only $316(10.3 \%$ (95\% CI $9.3 \%$ to $11.4 \%)$ ) of the patients.

\section{Cancers}

Optimal screening for malignancies, according to recommended guidelines, was performed in only 909 (23.9\%) of the patients for skin cancers, $608(26.7 \%)$ of the patients for colon cancer, $202(38.2 \%)$ of the patients for prostate cancer, $938(51.5 \%)$ of the patients for breast cancer, and 1383 (59.3\%) of the patients for uterine cancer.

\section{Osteoporosis}

Bone densitometry had been performed at least once in 2281 $(58.2 \%$ (95\% CI $56.6 \%$ to $59.7 \%)$ ) of the 3920 patients. Of all enrolled patients, 1733 (44.4\% (95\% CI 42.9\% to $46.0 \%)$ ) were receiving vitamin $\mathrm{D}$ supplementation at the time of the study visit.

Detailed listings by country of the percentage of patients optimally monitored for cardiovascular, infectious and cancer diseases are presented in online supplementary tables S10-S12.

\section{DISCUSSION}

This is the first population-based, cross-sectional observational study to assess multiple comorbidities and their management among a relatively large sample of patients with RA who were enrolled by rheumatologists in 17 participating countries on five different continents. This study confirms not only the relatively high prevalence of comorbidities among patients with RA, but also considerable intercountry variability in the prevalence of these comorbidities. ${ }^{29}$ It demonstrates that, at present, the management of comorbidities in patients with RA is far from optimal. As in this study, the systematic evaluation of RA patients for evidence of comorbidities may uncover previously undiagnosed conditions in some patients.

An important aim of this study was to evaluate the gap between current recommendations for detecting, managing and preventing comorbidities and their implementation in observed daily practice. To accomplish this objective, the scientific committee for the study created an a priori definition of optimal monitoring based largely on current recommendations provided by various international medical organisations. The optimal LDL cholesterol level on which the analysis of this study was based is that currently recommended by the French Ministry of Health, ${ }^{28}$ and this standard was applied to all study subjects in all participating countries regardless of local recommendations. However, for comorbidities such as cardiovascular disease, definitions were country-specific, and recommendations for monitoring or prevention varied slightly between participating countries. For example, the indication for initiation of antithrombotic drug prophylaxis was a history of a prior cardiovascular event in some countries and a $>20 \%$ risk of experiencing a cardiovascular event based on the Framingham Risk Score in others. ${ }^{27}$ Had this study used other standards for the detection, management and 
prevention of comorbidities, ${ }^{30} 31$ it might have found slightly different proportions. Regardless, this study demonstrates that compliance with recommended strategies is far from perfect and that this varies significantly among countries.

Although this study has numerous strengths, it also has several weaknesses. The comorbidities evaluated were selected for the study by the scientific committee and were not all-inclusive; some important comorbidities, such as tuberculosis, were not among those assessed. Despite the principal investigators in each country having been instructed to recruit rheumatologists working in different practice settings to enrol RA patients, it cannot be guaranteed that the prevalent cohort of 3920 patients studied here were fully representative of all RA patients in the participating countries. The study did not enrol RA patients from general practices who were not under the care of a rheumatologist. Moreover, some of the intercountry variability in the degree of RA disease activity observed in this study might reflect differences in the reason for which the patient was visiting the rheumatologist at the time of study participation: in some countries, patients are evaluated routinely even when their RA is under good control, whereas, in other countries, patients go to see their rheumatologist only when they experience a flare of disease activity. Also, cultural differences among patients recruited from different countries might lead to diverse interpretations of questions included in the questionnaire. Varied interpretation of the term 'depression' by subjects in different countries could account, in part, for the wide differences observed across countries in the prevalence of depression.

Several different types of bias are inherent in a prevalent cohort study of a chronic disease. The prevalence of some comorbidities might be overestimated because of diagnostic bias, in that patients with RA may more likely be offered screening for recognised comorbidities, or because of reporting bias, in that RA patients may more likely be diagnosed with comorbidities known to be associated with this inflammatory disease. The prevalence of other comorbidities might be underestimated because of truncation bias, in that RA patients with potentially life-threatening comorbidities may have been lost from the population before the cohort was enrolled. These biases may produce diverse effects in different countries. The lack of a comparator group without RA did not allow comparison in this study between the observed prevalence of comorbidities and their optimal management among RA patients with that in the general population or among patients with another disease state.

This study achieved its main objective, which was to evaluate and demonstrate intercountry variation in the detection, management and prevention of comorbidities among RA patients. It shows clear differences in the prevalence of certain risk factors, which might influence national policies regarding prevention strategies. For example, the high prevalence of tobacco smoking found among RA patients in the Netherlands (41.2\%) and Austria (47.5\%) might prompt targeted programmes to reduce this behaviour, which clearly predisposes not only to the development of RA, ${ }^{32}$ but also to cardiovascular disease ${ }^{33}$ and lung cancer, ${ }^{34}$ both of which are also significant comorbidities of RA. Other studies that have compared the prevalence of comorbidities among RA patients with those in the general population have shown a higher prevalence of cardiovascular events, ${ }^{8}$ infections, ${ }^{10} 11$ osteoporotic fractures $^{12}$ and lung cancer ${ }^{14}$ among RA patients. Nevertheless, the relatively large sample of RA patients who were enrolled from 17 countries on various continents allowed the present study to confirm the high prevalence of hepatitis in Asian ${ }^{35} 36$ and southern European countries ${ }^{37}$ and in Egypt. ${ }^{38} 39$
This study confirms the observation that monitoring of RA patients for cardiovascular risk is suboptimal ${ }^{19} 40-42$ and extends this assumption to other comorbidities. Moreover, it demonstrates that systematic assessment of RA patients for comorbidities facilitates the detection of abnormalities such as elevated blood pressure, hyperglycaemia and hypercholesterolaemia. These findings are in agreement with those of previous studies that suggested that cardiovascular risk factors are not optimally monitored and managed in 30-50\% of RA patients. ${ }^{40} 41$

Given the findings of the present study, the question arises as to how best to improve this situation. The treating rheumatologist should consider the periodic assessment of comorbidities as one of the tasks involved in treating a patient with RA. This should be carried out in collaboration with primary care providers and other specialists who are involved in the care of these patients. However, the increasing complexity of managing treatment of RA with effective combinations of traditional and biological DMARDs in the setting of progressively decreasing amounts of time available for direct interaction with the patient makes this additional responsibility challenging. The development and implementation of standardised programmes to detect, manage and prevent comorbidities in daily clinical practice, working in partnership with other healthcare providers such as nurses, ${ }^{42} 43$ might greatly facilitate the identification of and intervention to reduce the prevalence of comorbidities among patients with RA.

\section{Author affiliations}

${ }^{1}$ Medicine Faculty, Paris-Descartes University, Paris, France

${ }^{2}$ Rheumatology B Department, APHP, Cochin Hospital, Paris, France

${ }^{3}$ Department of Rheumatology, CHU Clermont-Ferrand, France

${ }^{4}$ Rheumatology Unit, Central Hospital "Dr Urquinaona", Maracaibo, Venezuela

${ }^{5}$ 3rd Rheumatology Department, National Institute of Rheumatology and

Physiotherapy, Budapest, Hungary

${ }^{6}$ Rheumatology Unit, La Paz University Hospital, Madrid, Spain

${ }^{7}$ Leeds Institute of Rheumatic \& Musculoskeletal Medicine, University of Leeds, UK

${ }^{8}$ NIHR Leeds Musculoskeletal Biomedical Research Unit, Leeds Teaching Hospitals NHS, UK

${ }^{9}$ Department of Rheumatology, Hospital Militar Central, Buenos Aires, Argentina

${ }^{10}$ Rheumatology Department, Charité University Medicine, Berlin, Germany

${ }^{11}$ Department of Rheumatology, Cairo University, Cairo, Egypt

${ }^{12}$ Rheumatology Department, El Ayachi Hospital, Salé, Morocco

${ }^{13}$ Department of Pharmacovigilance, Tokyo Medical and Dental University, Tokyo, Japan

${ }^{14}$ Department of Rheumatology, Allergy and Immunology, Chang Gung Memorial Hospital and Chang Gung University, Tao-Yuan, Taiwan

${ }^{15}$ Clínica Médica 1, Hospital Maciel, Universidad de la República, Montevideo,

Uruguay

${ }^{16}$ Department of Rheumatology, La Paz University Hospital, Madrid, Spain

${ }^{17}$ Division of Rheumatology, IRCCS policlinico S. Matteo foundation, University of Pavia, Pavia, Italy

${ }^{18}$ Institute of Infection, Immunity and Inflammation, College of Medical, Veterinary and Life Sciences, University of Glasgow, Glasgow, Scotland

${ }^{19}$ Division of Rheumatology, Department of Medicine 3, Medical University of Vienna, and 2nd Department of Medicine, Hietzing Hospital, Vienna, Austria

${ }^{20}$ Department of Rheumatology, Seoul National University Hospital, Seoul, Korea

${ }^{21}$ Department of Rheumatology and Clinical Immunology, Medisch Spectrum Twente and University of Twente, Enschede, The Netherlands

${ }^{22}$ Division of Infectious Diseases, Public Health and Preventive Medicine, Oregon Health and Science University, Portland, Oregon, USA

${ }^{23}$ Division of Rheumatology, Department of Medicine, University of Massachusetts Medical School and UMass Memorial Medical Center, Worcester, Massachusetts, USA

Correction notice This article has been corrected since it was published Online First. The affiliations have been corrected.

Acknowledgements The authors would like to thank all patients and investigators who participated in this study.

Contributors MD was the principal investigator for the study. Authors and study investigators gathered the data and interpreted the data. All authors reviewed and approved the report before submission. MD had full access to all of the data and had final responsibility for the decision to submit the manuscript for publication. 
Funding This study was conducted with the support of an unrestricted grant from Roche Ltd.

Competing interests None.

Patient consent Obtained.

Ethics approval Each appropriate body in the 17 participating countries.

Provenance and peer review Not commissioned; externally peer reviewed.

Open Access This is an Open Access article distributed in accordance with the Creative Commons Attribution Non Commercial (CC BY-NC 3.0) license, which permits others to distribute, remix, adapt, build upon this work non-commercially, and license their derivative works on different terms, provided the original work is properly cited and the use is non-commercial. See: http://creativecommons.org/licenses/by-nd3.0/

\section{REFERENCES}

1 Shourt CA, Crowson CS, Gabriel SE, et al. Orthopedic surgery among patients with rheumatoid arthritis 1980-2007: a population-based study focused on surgery rates, sex, and mortality. J Rheumatol 2012;39:481-5.

2 Salliot C, van der Heijde D. Long-term safety of methotrexate monotherapy in patients with rheumatoid arthritis: a systematic literature research. Ann Rheum Dis 2009;68:1100-4

3 Schoels M, Knevel R, Aletaha D, et al. Evidence for treating rheumatoid arthritis to target: results of a systematic literature search. Ann Rheum Dis 2010;69:638-43.

4 Wolfe F, Mitchell DM, Sibley JT, et al. The mortality of rheumatoid arthritis. Arthritis Rheum 1994;37:481-94.

5 Gabriel SE, Michaud K. Epidemiological studies in incidence, prevalence, mortality, and comorbidity of the rheumatic diseases. Arthritis Res Ther 2009;11:229.

6 Gabriel SE. Why do people with rheumatoid arthritis still die prematurely? Ann Rheum Dis 2008;67:30-4

7 Avina-Zubieta JA, Choi HK, Sadatsafavi M, et al. Risk of cardiovascular mortality in patients with rheumatoid arthritis: a meta-analysis of observational studies. Arthritis Rheum 2008;59:1690-7.

8 Lindhardsen J, Ahlehoff 0, Gislason GH, et al. The risk of myocardial infarction in rheumatoid arthritis and diabetes mellitus: a Danish nationwide cohort study. Ann Rheum Dis 2011;70:929-34.

9 Listing J, Gerhold K, Zink A. The risk of infections associated with rheumatoid arthritis, with its comorbidity and treatment. Rheumatology (Oxford) 2013;52:53-61.

10 Wotton CJ, Goldacre MJ. Risk of invasive pneumococcal disease in people admitted to hospital with selected immune-mediated diseases: record linkage cohort analyses. J Epidemiol Community Health 2012;66:1177-81.

11 Uresson C, Matteson EL. Malignancy as a comorbidity in rheumatic diseases. Rheumatology (Oxford) 2013;52:5-14.

12 Haugeberg G, Uhlig T, Falch JA, et al. Bone mineral density and frequency of osteoporosis in female patients with rheumatoid arthritis: results from 394 patients in the Oslo County Rheumatoid Arthritis register. Arthritis Rheum 2000;43:522-30.

13 Coulson KA, Reed G, Gilliam BE, et al. Factors influencing fracture risk, T score, and management of osteoporosis in patients with rheumatoid arthritis in the Consortium of Rheumatology Researchers of North America (CORRONA) registry. J Clin Rheumatol 2009;15:155-60.

14 Gullick NJ, Scott DL. Co-morbidities in established rheumatoid arthritis. Best Pract Res Clin Rheumatol 2011:25:469-83.

15 Liao KP, Solomon DH. Traditional cardiovascular risk factors, inflammation and cardiovascular risk in rheumatoid arthritis. Rheumatology (Oxford) 2013;52:45-52.

16 Solomon DH, Kremer J, Curtis JR, et al. Explaining the cardiovascular risk associated with rheumatoid arthritis: traditional risk factors versus markers of rheumatoid arthritis severity. Ann Rheum Dis 2010;69:1920-5.

17 Desai SS, Myles JD, Kaplan MJ. Suboptimal cardiovascular risk factor identification and management in patients with rheumatoid arthritis: a cohort analysis. Arthritis Res Ther 2012;14:R270.

18 Solomon DH, Karlson EW, Curhan GC. Cardiovascular care and cancer screening in female nurses with and without rheumatoid arthritis. Arthritis Rheum 2004;51:429-32.

19 Sowden E, Mitchell WS. An audit of influenza and pneumococcal vaccination in rheumatology outpatients. BMC Musculoskelet Disord 2007:8:58.
20 Kim SC, Schneeweiss S, Myers JA, et al. No differences in cancer screening rates in patients with rheumatoid arthritis compared to the general population. Arthritis Rheum 2012:64:3076-82.

21 van Assen S, Agmon-Levin N, Elkayam 0, et al. EULAR recommendations for vaccination in adult patients with autoimmune inflammatory rheumatic diseases. Ann Rheum Dis 2011;70:414-22.

22 Peters MJL, Symmons DPM, McCarey D, et al. EULAR evidence-based recommendations for cardiovascular risk management in patients with rheumatoid arthritis and other forms of inflammatory arthritis. Ann Rheum Dis 2010:69:325-31.

23 Arnett FC, Edworthy SM, Bloch DA, et al. The American Rheumatism Association 1987 revised criteria for the classification of rheumatoid arthritis. Arthritis Rheum, 1988;31:315-24.

24 Van der Heijde D, Van't Hof M, Van Riel P, et al. Development of a disease activity score based on judgment in clinical practice by rheumatologists. J Rheumatol, 1993;20:579-81.

25 http://www.HAS.org

26 http://www.rhumatismes.net

27 Grundy SM, Pasternak R, Greenland P, et al. Assessment of cardiovascular risk by use of multiple-risk-factor assessment equations: a statement for healthcare professionals from the American Heart Association and the American College of Cardiology. Circulation 1999;100:1481-92.

28 http://www.AFSSAPS

29 Naranjo A, Sokka T, Descalzo MA, et al. QUEST-RA Group. Cardiovascular disease in patients with rheumatoid arthritis: results from the QUEST-RA study. Arthritis Res Ther 2008; $10:$ R30.

30 Conroy RM, Pyörälä K, Fitzgerald AP, et al. SCORE project group. Eur Heart J 2003;24:987-1003.

$31 \mathrm{http}: / / w w w . r e y n o l d s r i s k s c o r e . o r g$

32 Boechat Nde O, Ogusku MM, Boechat AL, et al. Interaction between Smoking and HLA-DRB $1{ }^{*} 04$ Gene Is Associated with a High Cardiovascular Risk in Brazilian Amazon Patients with Rheumatoid Arthritis. PLoS One 2012:7:e4 1588.

33 Huxley RR, Yatsuya H, Lutsey PL, et al. Impact of age at smoking initiation, dosage, and time since quitting on cardiovascular disease in Africans americans and whites: the atherosclerosis risk in communities study. Am J Epidemiol 2012; 175:816-26.

34 Doll R, Peto R. Cigarette smoking and bronchial carcinoma: dose and time relationships among regular smokers and lifelong non-smokers. J Epidemiol Community Health 1978:32:303-13.

35 Zhong J, Gao YQ, Sun XH, et al. High prevalence of the B2+C2 subgenotype mixture in patients with chronic hepatitis B in Eastern China. Acta Pharmacol Sin 2012;33:1271-6.

36 Xiao J, Zhang J, Wu C, et al. Impact of hepatitis B vaccination among children in Guangdong Province, China. Int J Infect Dis 2012;16;e692-6.

37 Maio G, d'Argenio P, Stroffolini T, et al. Hepatitis C virus infection and alanine transaminase levels in the general population: a survey in a Southern Italian town. J Hepatol 2000;33:116-20.

38 Abdelwahab SF, Hashem M, Galal I, et al. Incidence of hepatitis C virus infection among Egyptian healthcare workers at high risk of infection. Clin Virol 2013:57:24-8.

39 Elgohry I, Elbanna A, Hashad D. Occult hepatitis B virus infection in a cohort of Egyptian chronic hemodialysis patients. Clin Lab 2012;58:1057-61.

40 Soubrier M, Zerkak D, Dougados M. Indications for lowering LDL cholesterol in rheumatoid arthritis: an unrecognized problem. J Rheumato/ 2006;33: 1766-9.

41 Gossec L, Salejan F, Nataf H, et al.; on behalf of the RHEVER rheumatology network. The challenges of cardiovascular risk assessment in the routine rheumatology outpatient setting. An observational study of 110 rheumatoid arthritis patients. Arthritis Care Res 2013;65:721-7.

42 Hebert PL, Sisk JE, Tuzzio L, et al. Nurse-led disease management for hypertension control in a diverse urban community: a randomized trial. J Gen Intern Med 2012;27:630-9

43 van Eijk-Hustings $Y$, van Tubergen $A$, Boström $C$, et al. EULAR recommendations for the role of the nurse in the management of chronic inflammatory arthritis. Ann Rheum Dis 2012:71:13-19. 